J. Product. \& Dev., 22(1): 103-119(2017)

\title{
In Silico DISCOVERY OF NOVEL HEPATITIS C VIRUS P7- TRANSACTIVATED PROTEIN1INHIBITOR BY USING STRUCTURE-BASED VIRTUAL SCREENING
}

Mahmoud M. El Hefnawi ${ }^{\text {* }}$; Mohamed E. Hasan ${ }^{2}$; Amal Mahmoud ${ }^{2}$;ehia A. Khidr ${ }^{3}$; El-Sayed A. El-Absawy and Alaa A. Hemeida ${ }^{2}$

${ }^{1}$ Informatics and Systems Department, Division of Engineering Research

Sciences, the National Research Centre, Egypt

${ }^{2}$ Bioinformatics Department, Genetic Engineering and Biotechnology

Research Institute, Sadat City University, Egypt

${ }^{3}$ Plant Biotechnology Department, Genetic Engineering and Biotechnology

Research Institute, Sadat City University, Egypt

Corresponding author:mahef@ aucegypt.edu, mahef111@gmail.com

\section{ABSTRACT}

Hepatitis $C$ Virus (HCV) infection is a serious cause of chronic liver disease worldwide with more than 170 million infected individuals at a risk of developing significant morbidity and mortality. Till date there is no effective drug for the treatment or vaccine to prevent this infection. The present study aims in discovering novel inhibitors which target an allosteric binding site of P7-transactivated Protein1 of HCV. Virtual screening uses computer-based methods to discover new ligands on the basis of biological structures. A structure based virtual screening of Zinc database by computational docking and the post docking analysis of energy calculations and interactions followed by ADMET studies were conducted. The approach adopted was receptorbased.

Docking screens, guided with contact pharmacophores and neural-network activity prediction models on all allosteric binding sites and MD simulations, constituted our analysis workflow for identification of potential hits. Steps included: 1) Using two phases docking screen with moe and Glide Xp programs, 2) Ranking based on scores, and important $H$ interactions. From the final hits, we selected best 10 compounds for further anti-HCV activity and cellular cytotoxicity assay. All 10 compounds have more potential to be 
considered as lead compoundsto inhibit ion channel activity of $p 7$ with docking score and binding energy (E_score) values ranging from 16.5087 to -15.8089 and all these compounds displayed no cellular cytotoxicity. Finally, 10 hit compounds of different scaffolds having interactions with important active site residues were predicted as lead candidates. These candidates having unique scaffolds have a strong likelihood to act as further starting points in the optimization and development of novel and potent $p 7$ ion channel inhibitors.

Keywords: In Silico Discovery, Novel Hepatitis C Virus P7Transactivated Protein1inhibitor, Using Structure-Based, Virtual Screening

\section{INTRODUCTION}

Hepatitis $\mathrm{C}$ virus (HCV) infection is a major cause of chronic liver disease. It is recognized as a major threat to global public health and according to the WHO estimates that a minimum of $3 \%$ of the world's population (more than 185 million people) is chronically infected with $\mathrm{HCV}$ around the world and 350 000-500 000 deaths estimated annually (Graham and Swan, 2015; Gower et al., 2014), because of chronicity, hepatic fibrosis, cirrhosis, and, increasingly, hepatocellular carcinoma (HCC) (Sugiyama, 2004).

The HCV genome consists of a 9.6-kb extraordinary variable positive strand RNA virus belonging to the genus Hepacivirus within the family Flaviviridae Simmonds, 2013)which encode a single polyprotein precursor. The later processed by both host and viral proteases into ten mature proteins (Niepmann, 2013; Moradpour and Penin, 2013).The p7-transactivated protein 1 is a small integral membrane protein of 127 amino acids with Mass (Da) 12,557, comprising viral proteins from several virus families which share the abilityto manipulate membrane permeability for ions by forming cation-selective pores (Montserret et al., 2010) and crucial forassembly and release of infectious virions (Gower et al., 2014).Overall, p7 appears to be a flexible protein as supported by the structural differences observed by various groups. Very important to note is the role of lipids or organic solvents that define the composition and thickness of the membrane thus influencing the final confirmation of 7 [(Chandler et al. 2012), (Radoicic et al. 2014)]. Models for hexameric or heptameric p7 channels from Gt1a, 1b and 2a have been obtained by molecular dynamics using either predicted or NMR-based 
structures of the corresponding monomers inserted into lipid bilayers (Cook et al. , 2013).

The RNA polymerase NS5B of Hepatitis C virus (HCV) is a wellcharacterised drug target with an active site and four allosteric binding sites. It takes too long and costs too much to develop a new drug. Therefore, drug repositioning efforts are gathering more attention (i.e., to screen an available drug for new uses). Currently, fifty plus drugs have been repositioned http://www.drugrepurposing.info/. Currently there is no vaccine available for HCV (Irshad et al., 2008). Current standard care of treatment for chronic hepatitis $\mathrm{C}$ is based on the combination of subcutaneous pegylated interferon$\alpha$ and oral nucleoside drug ribavirin. However, serious side effects and poor response rates render the development of novel anti-HCV therapy an urgent need (Neukam et al., 2009). Several clinical trials are currently progressing for specifically targeted antiviral therapies (STAT-C) inhibitors that target specific protein pockets to inhibit $\mathrm{HCV}$ functions, while additional trials proceed on compounds which target host cell proteins that the virus utilizes for its survival/replication (Kimet al., 2009).

In virtualscreening, large libraries of drug-like compounds that are commercially available are computationally screened again sttargets of known structure, and those that are predicted to bind well are experimentally tested $[1,2]$. However, database screening does not provide molecules that are structurally "novel" as these molecules have been previously synthesized by commercial vendors. Existing molecules can only be patented with a "method of use" patent covering their use for aunique application and not their chemical structure. In the denovo drug design approach, the 3D structure of the receptoris used to design structurally novel molecules that have never been synthesized before using ligand-growing programs and the intuition of the medicinal chemist[3].

Computer-aided drug discovery has recently had important successes: new biologically-active compounds have been predicted along with their receptor-bound structures and in several cases the achieved hit rates (ligands discovered per molecules tested) have been significantly greater than with HTS [1, 4-6]. Moreover, while it is rare to deliver lead candidates in the $\mathrm{nM}$ regime through VS, several reports in the recent literature describe the identification of $\mathrm{nM}$ leads directly from VS; these strategies will be discussed herein]7-9[. Therefore, computational methods play a prominentrole in the drug design and discovery process within the context of pharmaceutical research.In this review, we focus on the principles and applications of VS in 
the SBDD framework, starting from the initial stages of the process that include receptor and library preprocessing, to docking, scoring, and postprocessing of to pscoring hits. We also highlight several successful studies and protocols that led to $\mathrm{nM}$ leads, discuss novel applications of structureBased VS (SBVS) such as substrate identification for the discovery of novel metabolic pathways, and provide recent trends in library design. Limitations of SBVS are also examined. Finally, we present two developed VS protocols that aim to enhance inhibitor selectivity for the target protein structure.

Therefore, this study presents a workflow for virtual screening and its application to Drug Bank screening targeting the Hepatitis C Virus (HCV) p7-transactivated protein. Potential polypharmacological drugs are sought with predicted active inhibition on p7 ion channel. The approach adopted was receptor-based. Docking screens, guided with contact pharmacophores and neural-network activity prediction models on all allosteric binding sites and MD simulations, constituted our analysis workflow for identification of potential hits.

\section{MATERIALS AND METHODS}

\section{Virtual screening in structure-based drug discovery}

The general scheme of a SBVS strategy is shown in Figure (1), (Lavecchia and Di Giovanni, 2013). SBVS starts with processing the 3D target structural information of interest. The target structure may bederived from experimental data (X-ray, NMR or neutron scattering spectroscopy), homology modeling, or from Molecular Dynamics (MD) simulations. There are numerous fundamental issues that should be examined when considering a biological target for SBVS; for example, the druggability of the receptor, the choice of binding site, the selection of the most relevant protein structure, incorporating receptor flexibility, suitable assignment of protonation states, and consideration of water molecules in a binding site, to name a few. In fact, the identification of ligand binding sites on biological targets is becoming increasingly important. Another consideration for SBVS includes the careful choice of the compound library to be screened in the VS exercise according to the target in question, and the pre-processing of libraries in order to assign the proper stereochemistry, tautomeric, and protonation states.

Following library and receptor preparation, each compound in the library is virtually docked into the target binding site with a docking program. Docking aims to predict the ligand-protein complex structure by 


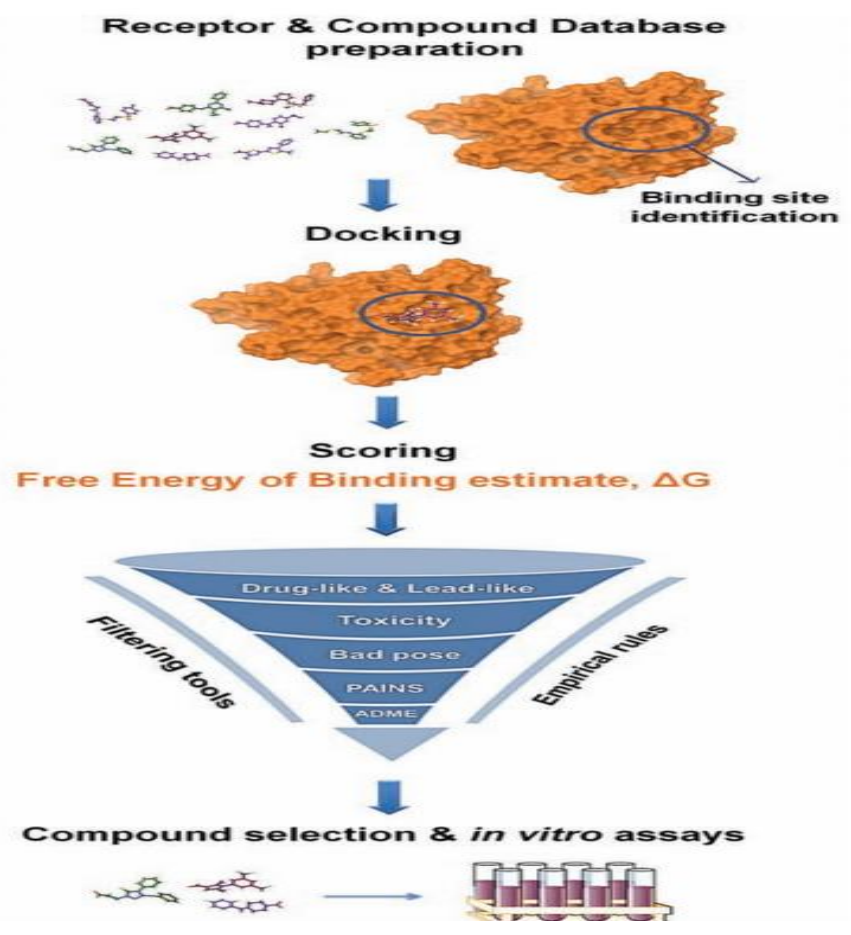

Figure (1). Structure-Based Virtual Screening work-flow.

exploring the conformational space of the ligands within the binding site of the protein. A scoring function is then utilized to approximate the free energy of binding between the protein and the ligand in each docking pose. Docking and scoring produce ranked compounds, which are then post-processed by examining calculated binding scores, validity of generated pose, undesirable chemical moieties, metabolic liabilities, desired physicochemical properties, lead-likeness, and chemical diversity. Post-processing results in a small number of selected compounds, which proceed to experimental assaying (Cheng et al., 2013).

\section{Virtual screening using Zinc database}

The ZINC database is a curated collection of commercially available chemical compounds prepared especially for virtual screening [15]. ZINC is used by investigators (generally people with training as biologists or chemists) in pharmaceutical companies, biotech companies, and research universities. There are many subsets available in the Zinc database. We had 
selected leads now subset containing 1,283,469 molecules which are kept ready to perform virtual screening.

\section{ADME Prediction (Absobtion, Distribution, Metabolism, Excretion)}

Predicting physiochemical properties of a chemical compound will always cut short the expensive experimental testing and hard labour. Molecular Docking studies revealed that 10 compounds from the Zinc small molecule databank has potential binding affinity towards HCV NS5B RdRp. Hence, the ADME predictions of these compounds was carried out using freely and commercially available web based ADME Boxes developed by Pharmaco Algorithms (http://pharmaalgorithms.com/webboxes/). It is a software module that calculates physiochemical properties, oral availability (human), human intestinal absorption, plasma bound distribution based on the chemical structure.

\subsection{Protein Preparation Schemes for SBVS}

The success of a SBVS campaign largely depends on reasonable starting structures for both the protein and the ligand. A typical PDB structure file consists only of heavy atoms (if the input is an X-ray structure) and may contain water molecules, cofactors, activators, ligands, and metal ions as well as several protein subunits. The general proposed strategy is to first determine the protonation states of the amino acids in the protein using available software. Popular freely available software includes PROPKA [13], H++ [14], SPORES (Brink and Exner, 2010). The next step is to assign hydrogen atoms and optimize protein hydrogen bonds according to an optimal hydrogen bond network. A widely-used software for these tasks is the PDB2PQR software (Dolinskyet al., 2007). The next steps are assignment of partial charges, capping of residues, treating metals, filling in missing loops and missing side chains, and minimizing the protein structure to relieve steric clashes.

\subsection{Binding Site Identification}

Binding site identification is often an additional prerequisite for performing SBVS, when the binding site is not known or when new, allosteric modulators of protein function are sought. Ideally, the target binding site is a pocket, typically a concave, having a variety of probable hydrogen bond donors and acceptors and hydrophobic characteristics. Examples of such an approachinclude SiteMap (Schrodinger 2013), FTMap 
(Ngan et al., 2012), Fpocket (le Guilloux et al., 2009) and MDpocket (Schmidtke et al., 2011).

\subsection{Compound Database Preparation}

The construction of compound databases is the next important step in the SBVS process. Databases for SBVS contain drug-like small molecules, often freely available or available via purchase or synthesis, which possess desirable characteristics such as stability and solubility in aqueous media.

Several rules state that drug-like compounds should have molecular weight lower than 500, lipophilicity $(\log \mathrm{P})$ lower than 5 , less than five hydrogen bond donors, and less than 10 hydrogen bond acceptors. Molecules may be considered as drug-like within a range of $\log \mathrm{P}$ in 0.4 to +5.6 , molar refractivity from 40 to 130, molecular weight from 180 to 500, number of atoms from 20 to 70 (includes H-bond donors (e.g.; OH's and NH's) and H-bond acceptors (e.g.; N's and O's), polar surface area no greater than $140 \AA 2$, and/or fewer than ten rotatable bonds (Veber et al., 2002). Chembio server is a publicly available online application specializing in filtering and selection of small molecules (Athanasiadis et al., 2012). The objective of this application is to facilitate compound preparation prior to (or after) VS computations by utilizing its many sections, such as (i) basic search, (ii) filtering (steric clashes and toxicity), (iii) advanced filtering based on custom chosen physicochemical properties, (iv) clustering (according to structure and compound physicochemical properties providing representative compounds for each cluster), (v) customized pipeline and (vi) visualization of compound' properties through property graphs and thus, increase the efficiency and the quality of compounds that proceed to in vitro assaying.

\subsection{Docking \& Scoring}

A large number of docking programs have been developed recently, including AutoDock (Morris et al., 2009), Dock (Ewing et al., 2001), FlexX (Rarey et al., 996), Glide (Friesner et al., 2004). Docking entails predicting the protein-ligand complex structure and is followed by scoring in SBVS in order to rank the compounds. Docking programs utilize various methods of conformational search in order to explore the ligand conformational space; these are categorized as following: a) Systematic methods, which place ligands in the predicted binding site after considering all degrees of freedom, b) Random or stochastic torsional searches about rotatable bonds, such as Monte Carlo and genetic algorithms to "evolve" new low energy conformers, 
(c) Molecular Dynamics simulation methods and energy minimization for exploring the energy landscape of a molecule. In order to rank compounds, docking programs utilize scoring functions that aim to estimate the free energy of binding of a ligand to a specific target based on a generated docked pose after docking different ligands of a database. Commonly-used scoring functions can be categorized as follows: (a) Force field-based functions that estimate the binding free energy by summing the strength of intermolecular van der Waals, electrostatic interactions and hydrogen bonding between all atoms of the two binding partners in the complex. (b) Empirical scoring functions that are based on counting the number of various types of interactions between the two binding partners, i.e. hydrophobic contacts, number of hydrogen bonds and number of rotatable bonds immobilized in complex formation.These functions have proven to be successful for many protein-ligand complexes. (c) Knowledge-based functions that use statistical observations of intermolecular contacts in receptor-ligand complexes with known structural conformations.

\subsection{Improving pose/compound selection after docking (post-processing)}

Visual inspection of thousands of docking poses is normally needed by the medicinal chemist in order to select the appropriate compound set for assaying. To this end, significant efforts have been dedicated to increase the efficiency and the quality of compound selection (Waszkowycz, 2009). These tools may be also used for post-processing of SBVS results are readily available. The ChemBio Server for example, uses vdW filtering to remove compounds with steric clashes. Poses that are far from the energy minimum are unlikely to be adopted in nature and hence, should be discarded. Compounds that pass vdW filtering may be then subjected to more stringent physicochemical property filtering compared to the initial compound selection for SBVS. Subsequently, hierarchical clustering may be performed in order to group compounds with similar structures/physicochemical properties and derive subsets with maximal chemical diversity (Athanasiadis et al., 2012).

\subsection{Binding energy and binding affinity calculations}

To identify the most potential ligands, binding affinities of thehitsNS3/4A protease complexes were calculated with generalized Born / volume integral (GB / VI) implicit solvent method implemented in MOE [39]. Generalized Born interaction energy is the non-bonded interaction energy between the receptor rmolecule and the ligand that includes Coulomb 
electrostatic interaction, Vander Waals, and implicit solvent interaction energies. The strain energies of ligands and receptor molecules are, however, not taken into account. During calculation solvent molecules were ignored. The estimated binding affinity is that of the London $\mathrm{dG}$ scoring function reported in unit of Kcal/Mol. During calculations the atoms of the receptor molecule away from the ligand were kept rigid while receptor atoms in the locality of the ligand (in the binding site) were kept flexible but were subjected to tether restraints that discourage gross movement. The ligand atoms were set free to move at the binding pocket. In each case an energy minimization of binding pocket inNS3/4Aprotease-ligand complex was performed before calculating binding affinity. The binding affinity was calculated for each hit after energy minimization, and reported in unit of Kcal/Mol.

\section{RESULT AND DISCUSSION}

\section{3-D Structure prediction}

Different bioinformatics servers were used to predict and analyze the p7-transactivated protein 1 and to discover protein binding motifs relating to its hubness, promiscuity and biological functions. Also the study included the prediction of family conserved regions, secondary structure, solvent accessibility, tertiary structure, interaction motifs, post-translational modification sites and disordered regions. A fairly best model had been obtained from I-TASSER server after refinement and energy minimization according to best value of C-score, RMSD, TM-score and QMEAN Z-score as shown in fig.2. P7 has two Post-translational modification sites prediction: Camp_phospho_site and Myristyl site which has ion channel/pore-like function and crucial for production and release of infectious HCV particles from infected cells.

\section{Drug-likeliness and drug-Score}

Drug-likeness is an important parameter because drug-like molecules exhibit favorable absorption, distribution, metabolism, excretion, toxicological (ADMET) parameters. Currently, there are many approaches to a ssessa compound drug-likeness based on topological descriptors, fingerprints of molecular drug-likeness structure keys or other properties such as clog $\mathrm{P}$ and MW. In this study, moe program was used for calculating the fragment based drug-likeness of the lead compounds and comparing them with MK-5172. 


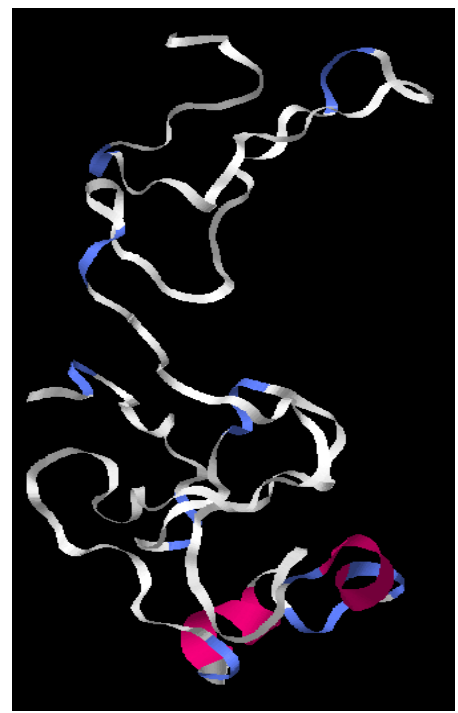

(a)

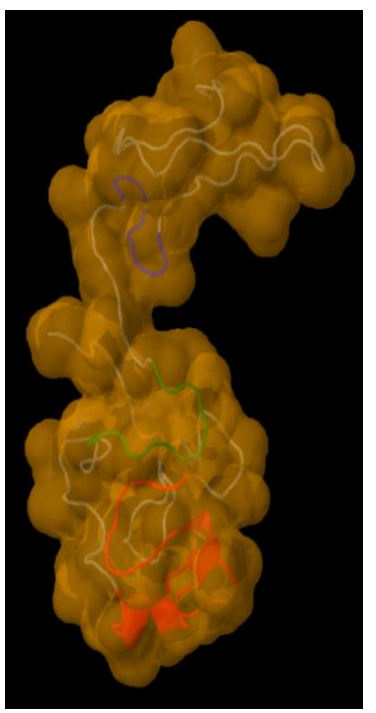

(b)

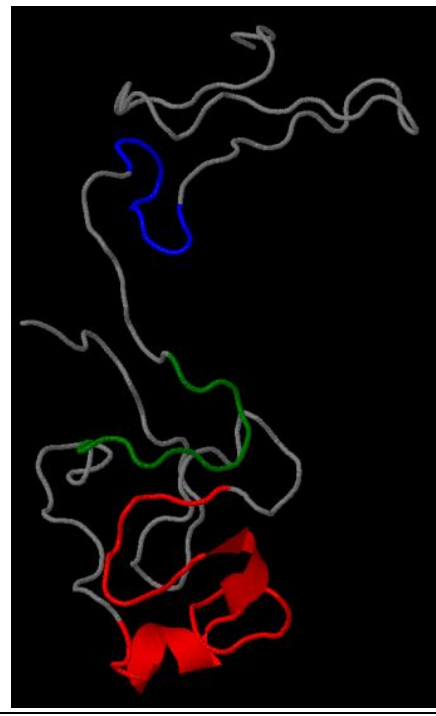

(c)

Figure 2: The best predicted three-dimensional structure ofp7 protein by I-TASSER server. (a) Ribbon view of best predicted model, (b) Solvent-accessible surface view shows the exposed regions. (c)The cartoon view shows known and predicted motifs: The Asp/Glu (IPB006034A, 32-39) was highlighted in blue, CTNNB1-bd-N (IPB013558C, 48-57) was highlighted in green, Asp/Gls (IPB006034C, 71-100) was highlighted in red and the rest of predicted model was highlighted in gray.

The drug score combines drug-likeliness, miLogP, $\log \mathrm{S}, \mathrm{MW}$, and toxicity risks in one convenient value than may be used to judge the compound's overall potential to qualify for a drug.

\section{Molecular docking}

To further refine the hit compounds, all the initially retrieved hits were docked into the binding site of NS3/4A protease using the docking protocol implemented in MOE. Before docking the initial hits, the ligand from the complex structure was extracted and re-docked into the binding cavity of protein to validate the docking protocol. The root mean square deviation (RMSD) between the co-crystallized and re-docked conformation was calculated by using SVL script of MOE and found to be equal to $2.07 \mathrm{~A}^{\circ}$, suggesting that our docking protocol is reliable in reproducing the experimentally determined binding mode for corresponding protein-ligand complex. The MOE docking protocol and the parameters set could be used to 
search the binding modes of other compounds accordingly. Using the same docking protocol all the initial hits were docked into the binding pocket ofHCV p7 protein. A maximum of 10 conformations were allowed to be saved for each ligand using the default parameters of MOE already discussed. The top ranked conformations of alldocked compounds were saved in a separate database. On the basis of docking score, 300 top ranked compounds from lead-like zinc database were selected for further evaluation. The resulted binding interactions between these 3.4 million hits and protein were visually observed using LigPlot implemented in MOE and those molecules which revealedsignificant interactions with most of the important binding pocket residues (VAL47, GLY49, GIN50, PRO51, GLY52, PRO53, HIS54, LEU58, ARG91, LEU99, TRP100, THR101, PRO102, GLY104, SER105, ALA106, ALA109, PRO110, THR120, THR121, SER123)of p7 protein were selected as promising hits. Among these 3.4 million compounds, 30 showed crucial interactions with the important residues of target protein. These 30 compounds were further subjected to Binding energy and Binding affinity calculation.

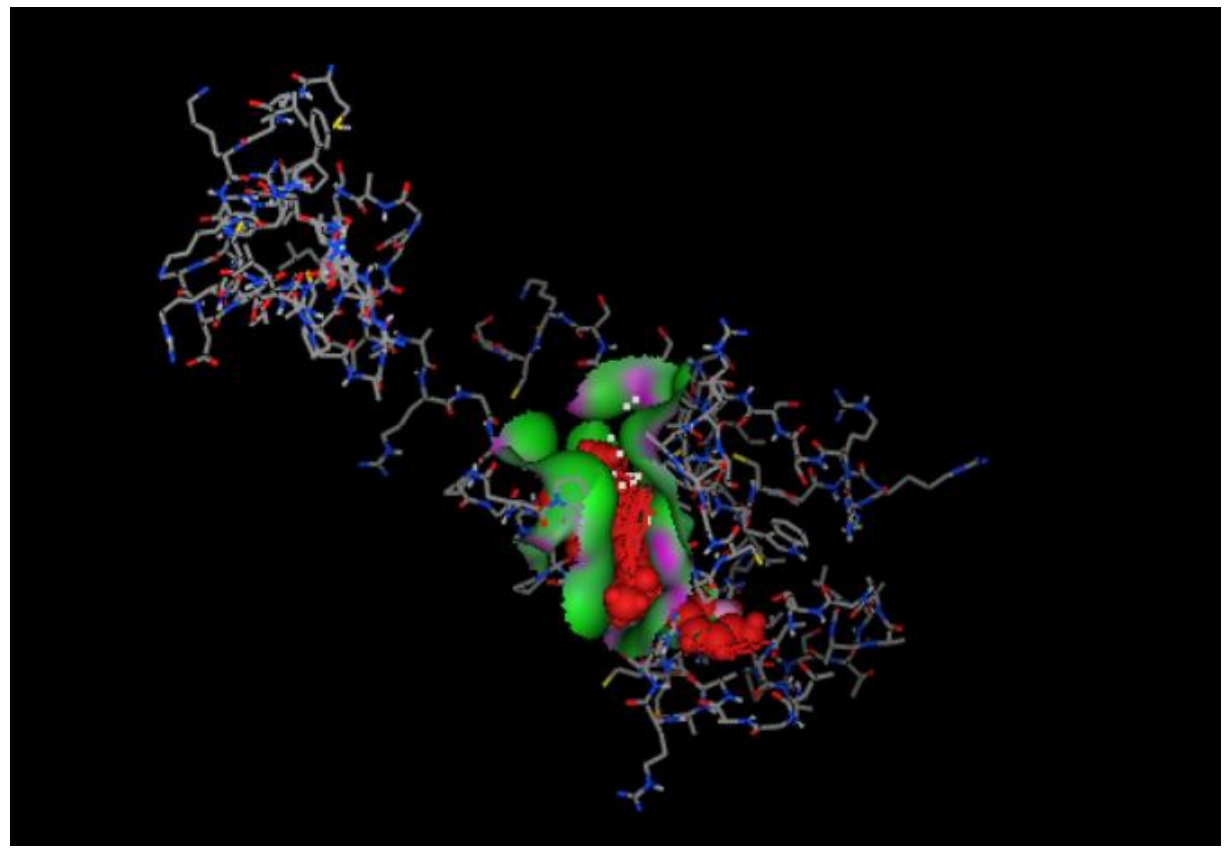

Figure 3. Complex structure of important binding pocket residues of $\mathrm{p} 7$ protein with ligand. 


\section{Binding Energy and Binding Affinity Calculations.}

To identify the most potential ligands, binding affinities for all the 30 compounds including ligand of the complex structure were calculated with generalized Born/volume integral (GB/VI)implemented in MOE. In each case an energy minimization of binding pocket in $-\mathrm{p} 7$ protein ligand complex was performed before calculating binding affinity. The binding affinity was calculated for each hit after energy minimization, and reported in unit of $\mathrm{Kcal} / \mathrm{Mol}$. The selection criteria for the most promising candidates were, compounds having binding energy and binding affinity good or equal to that calculated for there ference ligand in the complex structure, visualization of each hit in the binding cavity and the selection of only those hits showing interactions with important residues in binding cavity of $\mathrm{HCV}$ p7 protein. Applying the above mentioned criteria, selected 10compoundswith lead-like zinc database ID (ZINC-19680637, ZINC-03907796, ZINC-05357579, ZINC-03907795， ZINC-69535946， ZINC-78563224， ZINC-13184194, ZINC-40511048, ZINC-57738555 and ZINC-96185821 were ranked according to docking score and binding energy (E_score)values ranging from -16.5087 to -15.8089 (Table 1). The binding mode, binding affinity, binding energy and visual prediction showed that these predicted lead compounds might act as novel, potent and structurally diverse inhibitors of ion channel of HCV p7 protein. The 2D structures of these retrieved hits are shown in Figure 4.

Table 1: ZINC database ID, Docking Scores, binding energies, binding affinities and mseq of hit compounds.

\begin{tabular}{|c|c|c|c|c|c|}
\hline Compound & $\begin{array}{c}\text { ZINC databse } \\
\text { ID }\end{array}$ & $\begin{array}{c}\text { Docking } \\
\text { score } \\
\text { (S ) }\end{array}$ & $\begin{array}{c}\text { Binding } \\
\text { affinity } \\
\text { Kcal/Mol }\end{array}$ & $\begin{array}{c}\text { Binding } \\
\text { energy } \\
\text { Kcal/Mol }\end{array}$ & mseq \\
\hline 1 & 19680637 & -16.5087 & -10.9135 & -13.1368 & 2786 \\
\hline 2 & 03907795 & -16.4606 & -10.7436 & -20.2652 & 1024 \\
\hline 3 & 05357597 & -16.217 & -11.0642 & -16.204 & 2905 \\
\hline 4 & 03907795 & -16.1437 & -10.6818 & -17.8424 & 1024 \\
\hline 5 & 69535946 & -16.0993 & -11.4105 & -20.3808 & 3409 \\
\hline 6 & 78563224 & -16.0092 & -11.732 & -21.0525 & 3968 \\
\hline 7 & 13184194 & -15.8864 & -11.3873 & -17.1745 & 1853 \\
\hline 8 & 40511048 & -15.8343 & -10.8435 & -17.389 & 574 \\
\hline 9 & 57738555 & -15.8328 & -10.8615 & -14.9934 & 1109 \\
\hline 10 & 96185821 & -15.8089 & -10.2288 & -17.1352 & 1809 \\
\hline
\end{tabular}



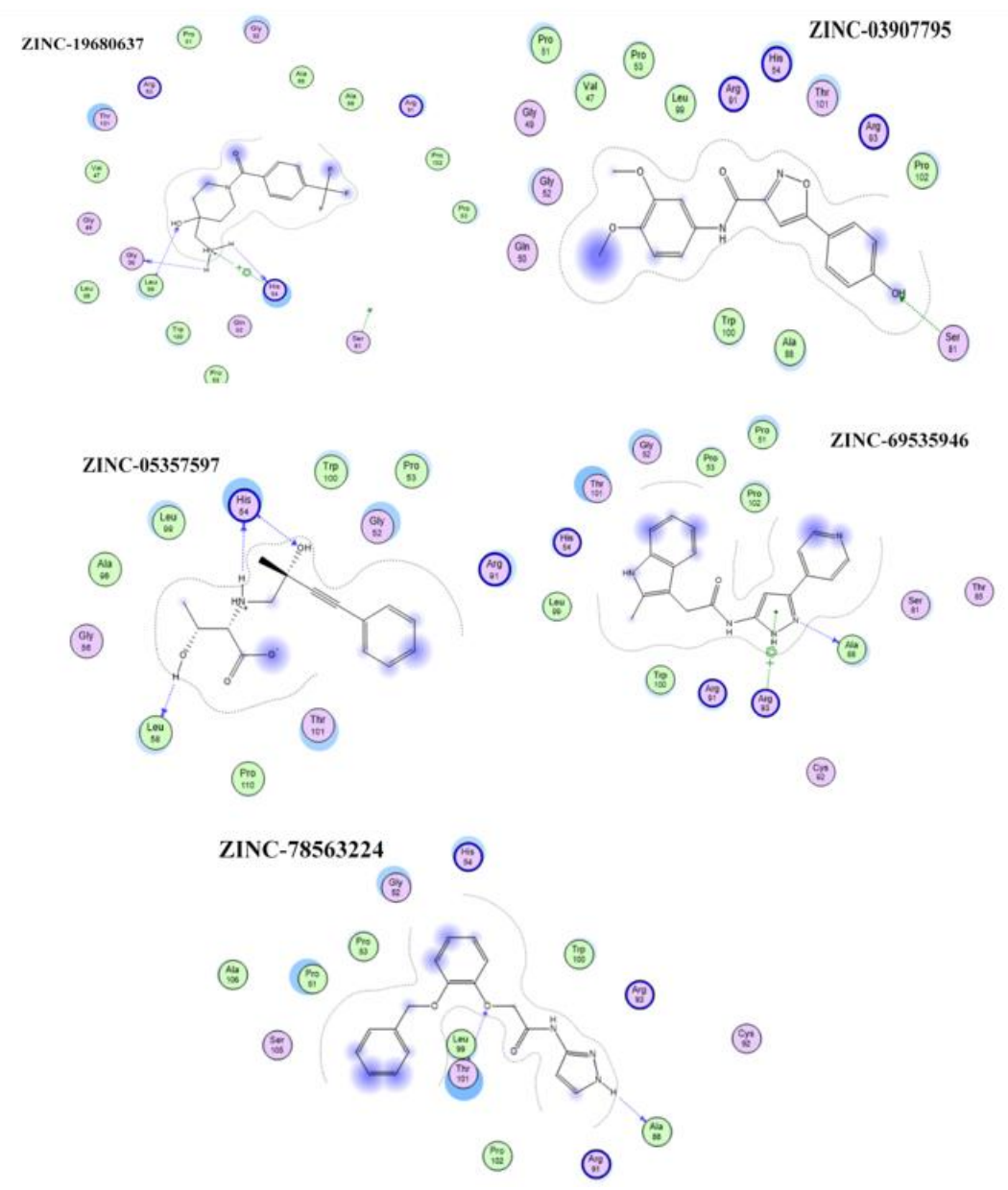

Figure 4.Docking and interaction of best ligand retrieved from ZINC database with target protein

\section{Conclusion}

In this study, the development of a chemical feature based 3D docking model of HCV p7 inhibitors have been described from the crystal structure of p7 inhibitors in complex with a macrocyclic inhibitor interacting with both target predicted $\mathrm{p} 7$ protein via MOE and Glide XP docking constructing tool. The developed docking hypothesis consisted of seven key features including four hydrogen bond acceptors (Acc), one hydrophobic (Hyd), one for lone pair or active hydrogen (Atom L) and a heavy atom feature (Atom Q). A 
structure based virtual screening of Zinc database by computational docking and the post docking analysis of energy calculations and interactions followed by ADMET studies were conducted. The approach adopted was receptor-based. Docking screens, guided with contact pharmacophores and neural-network activity prediction models on all allosteric binding sites and MD simulations, constituted our analysis workflow for identification of potential hits. From the final hits, we selected best 10 compounds for further anti-HCV activity and cellular cytotoxicity assay.

All 10 compounds have more potential to be considered as lead compounds to inhibit ion channel activity of $\mathrm{p} 7$ with docking score and binding energy (E_score) values ranging from -16.5087 to -15.8089 and all these compounds displayed no cellular cytotoxicity. Finally, 10 hit compounds of different scaffolds having interactions with important active site residues were predicted as lead candidates. These candidates having unique scaffolds have a strong likelihood to act as further starting points in the optimization and development of novel and potent $\mathrm{p} 7$ ion channel inhibitors.

\section{REFERENCES}

Athanasiadis, E.; Cournia, Z.; Spyrou, G. ChemBio Server: A web based pipeline for filtering, clustering and visualization of chemical compounds used in drug discovery. Bioinformatics, 2012, 28, (22): 3002-3003.

Chandler, D.E.; Penin, F.; Schulten, K.; Chipot, C. The p7 protein of hepatitis $\mathrm{C}$ virus forms structurally plastic, minimalist ion channels. PLoS. Comput. Biol.,, 2012, 8, e1002702.

Cheng, T.; Li, Q.; Zhou, Z.; Wang, Y.; Bryant, S.H. Structure based virtual screening for drug discovery: a problem-centric review. AAPS J., 2012, 14, (1): 133-141.

Cook, G.A.; Dawson, L.A.; Tian, Y.; Opella, S.J. Three-dimensional structure and interaction studies of hepatitis $\mathrm{C}$ virus p7 in 1,2dihexanoyl-sn-glycero-3-phosphocholine by solution nuclear magnetic resonance. Biochemistry, 2013, 52: 5295-5303.

Dolinsky, T.J.; Czodrowski, P.; Li, H.; Nielsen, J.E.; Jensen, J.H.; Klebe, G.; Baker, N.A. PDB2PQR: Expanding and upgrading automated preparation of biomolecular structures for molecular simulations. Nuc. Acids Res., 2007, 35: W522-W525. 
Ewing, T.J.; Makino, S.; Skillman, A.G.; Kuntz, I.D. DOCK 4.0: search strategies for automated molecular docking of flexible molecule databases. J. Comput. Aided. Mol. Des., 2001, 15:411-428.

Friesner, R.A.; Banks, J.L.; Murphy, R.B.; Halgren, T.A.; Klicic, J.J.; Mainz, D.T.; et al. Glide: A new approach for rapid, accurate docking and scoring. 1. Method and assessment of docking accuracy. J. Med. Chem., 2004, 47: 1739-1749.

Gower, E., Estes, C., Blach, S., Razavi-Shearer, K., Razavi, H. Global epidemiology and genotype distribution of the hepatitis $\mathrm{C}$ virus infection. J. Hepatol, 2014, 61(1): 45-57.

Graham, C.S., Swan, T. A path to eradication of hepatitis C in low- and middle income countries. Antiviral Res., 2015; 119: 89-96.

Irshad M, Khushboo I, Singh S. Hepatitis C virus (HCV): a review of immunological aspects. Int. Rev. Immunol., 2008, 27 (6): 497-517.

Kim SI, Shin D, Lee H, Ahn BY, Yoon Y, Kim M. Targeted delivery of siRNA against hepatitis $\mathrm{C}$ virus by apolipoprotein A-I-bound cationic liposomes. J. Hepatol., 2009, 50 (3): 479-488.

Lavecchia, A.; Di Giovanni, C. Virtual screening strategies in drug discovery: a critical review. Curr. Med. Chem., 2013, 20, (23): 28392860.

le Guilloux, V.; Schmidtke, P.; Tuffery, P. Fpocket: An open source platform for ligand pocket detection. BMC Bioinformatics, 2009, 10: 168.

Ma XH, Shi Z, Tan C, Jiang Y, Go ML, Low BC, Chen YZ: In-silico approaches to multi-target drug discovery: computer aided multi-target drug design, multi-target virtual screening. Pharmaceutical Research, 2010, 27 (5): 739-749.

Montserret, R., Saint, N., Vanbelle, C., Salvay, A.G., Simorre J.P., Ebel, C., Sapay, N., Renisio, J.G., Böckmann, A., Steinmann, E., Pietschmann, T., Dubuisson, J., Chipot, C., Penin, F. NMR structure and ion channel activity of the $\mathrm{p} 7$ protein from hepatitis $\mathrm{C}$ virus. J. Biol. Chem. , 2010, 285: 31446-31461.

Moradpour, D., Penin, F. Hepatitis C virus proteins: From structure to function. Curr. Top. Microbiol. Immunol., 2013; 369: 113-142.

Morris, G.M.; Huey, R.; Lindstrom, W.; Sanner, M.F.; Belew, R.K.; Goodsell, D. S.; Olson, A. J. Autodock4 and AutoDockTools4: automated docking with selective receptor flexiblity. $J$. Comput. Chem., 2009, 16: 2785-2791. 
Neukam K, Macias J, Mira JA, Pineda JA: A review of current anti-HCV treatment regimens and possible future strategies. Expert Opin Pharmacother., 2009, 10 (3): 417-433.

Ngan, C.H.; Bohnuud, T.; Mottarella, S.E.; Beglov, D.; Villar, E.A.; Hall, D.R.; Kozakov, D.; Vajda, S. FTMAP: extended protein mapping with user-selected probe molecules. Nuc. Acids Res., 2012, 40, (Web Server Issue), W271-W275.

Niepmann, M. Hepatitis C virus RNA translation. Curr. Top. Microbiol. Immunol., 2013; 369: 143-166.

Radoicic, J.; Lu, G.J.; Opella, S.J. NMR structures of membrane proteins in phospholipid bilayers. Q. Rev. Biophys., 2014, 47: 249-283.

Rarey, M.; Kramer, B.; Lengauer, T.; Klebe, G.: A fast flexible docking method using an incremental construction algorithm. J. Mol. Biol., 1996, 261 , (3): 470-489.

Schmidtke, P.; Bidon-Chanal, A.; Luque, F.J.; Barril, X.: MDpocket: open-source cavity detection and characterization on molecular dynamics trajectories. Bioinformatics, 2011, 27, (23): 3276-3285.

Simmonds, P.: The origin of hepatitis C virus. Curr. Top. Microbiol. Immunol., 2013; 369: 1-15.

Site Map, Schrodinger, LLC, 2013.

Sugiyama, K.: Genomic structure and function of untranslated region, structural region and nonstructural region of hepatitis $\mathrm{C}$ virus RNA. Nippon Rinsho., 2004; 62 (7): 32-37.

Ten Brink, T.; Exner, T.E.: pKa Based Protonation States and Microspecies for Protein-Ligand Docking. J. Comput. Aided Mol. Des., 2010, 24: 935-942

Veber, D.F.; Johnson, S.R.; Cheng, H.Y.; Smith, B.R.; Ward, K.W.; Kopple, K.D.: Molecular properties that influence the oral bioavailability of drug candidates. J. Med. Chem., 2002, 45, (12): 2615-2623.

Waszkowycz, B.: Towards improving compound selection in structure-based virtual screening. Drug Discov. Today, 2008, 13, (5-6): 219-226

Wilhelm S, Carter C, Lynch M, Lowinger T, Dumas J, Smith RA, Schwartz B, Simantov R, Kelley S: Discovery and development of sorafenib: a multikinase inhibitor for treating cancer. Nature Reviews Drug discovery., 2006, 5 (10): 835-844. 


\section{الاكتشاف النظري لمثبطات عمل ووظيفة بروتين بي سبعة للفيروس

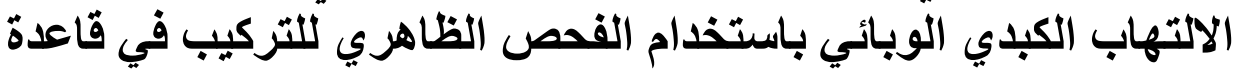 بيانات المركبات الفئد}

محمود الحفناوي، محمد حسن، آمال محمود، يحيي خضر، السيد العبساوي،

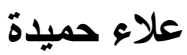
معهد الهندسة الور اثية ـ جامعة المنوفيه ـ مدينة السادات.

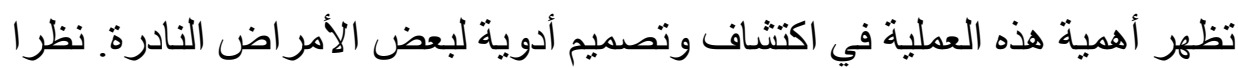

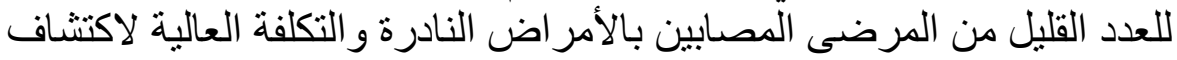

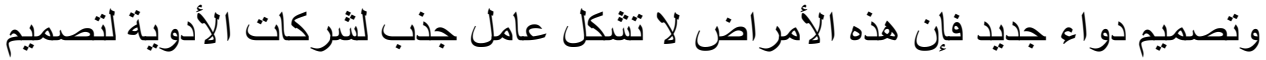

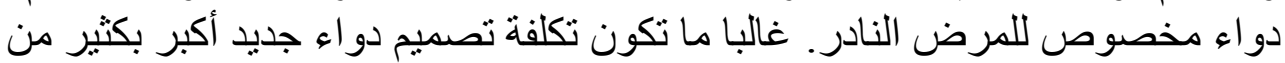
العائد المادي المتوقع من بيع وتسويق هذا الدواء.

يتم تجاوز هذه المشكلة بما يعرف بإعادة توصيف الأدوية و التي تلعب المعلوماتية

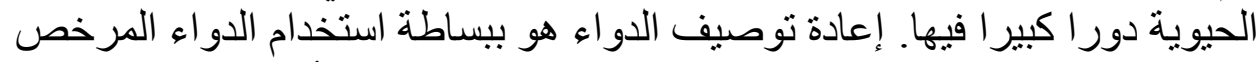

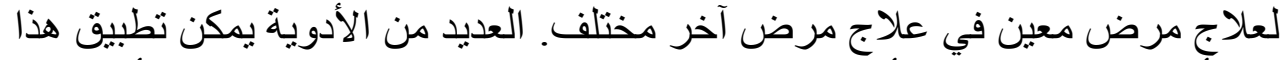

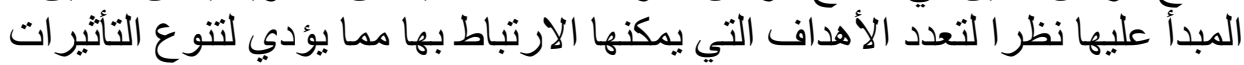

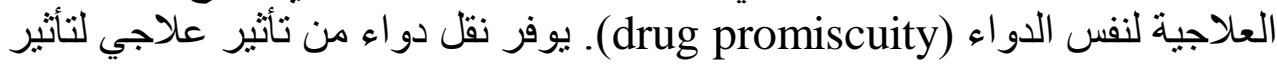

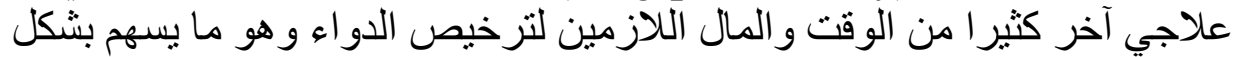

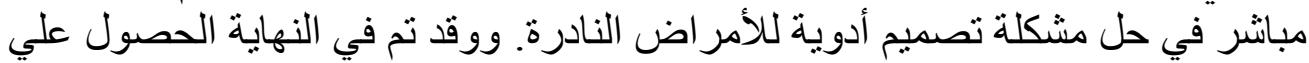

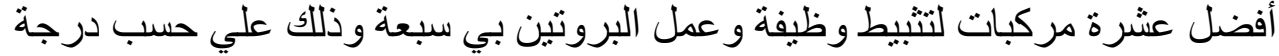

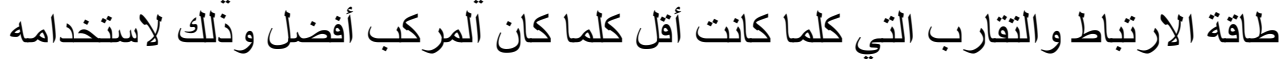

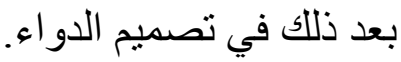

